\title{
Design and Implementation of Electronically Controlled On off Prism based on WSNs
}

\author{
Wei Guo ${ }^{1}$, Yun Bai ${ }^{1}$, Peng Zhang ${ }^{1}$ and Xiaolu Liu ${ }^{2}$ \\ ${ }^{1}$ Shanghai Medo Monitoring Technology Co., Ltd., Zhangheng Road, Shanghai, China \\ ${ }^{2}$ Shanghai Urban Construction Design and Research Institute, Dongfang Road, Shanghai, China
}

\begin{abstract}
Aiming at the problem of multiple prisms recognition in a narrow field angle in the application of TPS automatic measurement, a novel prism node is designed and implemented. Based on WSNs, measuring prism and electrically controlled dimming glass are grouped together as a prism node. It can easily switch between the fuzzy or the transparent state, to help TPS search and observe the correct prism automatically. Its feasibility is verified by experiments, which greatly simplifies the complexity of engineering installation and operation.
\end{abstract}

\section{Introduction}

With the development of measurement devices, Total Station Position System (TPS) integrated electro-motor and embedded an automated software become (Wang system based on Parer ang et al., 2017; Paraforos et al., 2017). It can automatically target recognition (ATR), automatic aiming, automatic angle measurement, automatic recording, working like a robot, observing hundreds of prisms continuously and repeatedly in construction surveying and deformation monitoring application (Gumilar et al., 2017).

But in automatic measurement applications, because of the positional relation between monitoring point and TPS station, multiple targets often appear in a narrow field angle, and it make TPS can not recognize the right prism and lead to an error result(Xiong et al., 2016). Aiming at this problem, some strategic processing methods are presented, but they can't solve this problem effectively. Sokkia and Leica automatic TPS can check out how many prisms in a field angle, and observe the nearest one as the result acquiescently, but in most cases this strategy maybe not get the right position of the right prism in automatic monitoring applications. Some automatic monitoring software can detect whether the measurement data is correct by calculating distance or coordinates, but it can not help TPS find the right prism once the mistake occurs. Trimble invent a kind of active switch prism which combined a common prism with a lens hood, and using an electric motor control the lens hood up or down. This device can solve the prism recognition problem in some application cases, but due to motor power consumption is larger, power supply cable and wired control are necessary, this restricts the convenience of engineering applications and is not suitable for outdoor monitoring and long distance applications.

To solve the problem, a kind of on-off prism node based on WSNs is designed and implemented. This node control whether the electrically controlled dimming glass is electrified, and then control whether the prism is in a transparent state. It avoids the measuring errors caused by multiple prisms in a narrow field angle. The feasibility of the prism node is verified by experiments, and the influence of dimming glass on measurement accuracy is analysed, onsite application conditions and installation methods are also given.

\section{On-Off Prism Design Based On WSNs}

\subsection{Choose a Suitable Network Platform for On- off Prism Node}

In order to meet engineering conditions, the wireless network platform of prism node should meet the following technical conditions:

(1) Wireless ad-hoc network communication;

(2) Stable and reliable communication mechanism, intelligent network expansion;

(3) Big communication radius, strong anti-interference ability, and good diffraction performance;

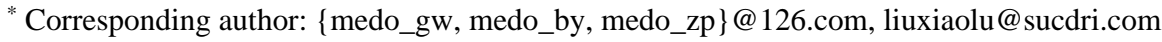


(4) Support embedded application implants in order to embed measurement control management and data preprocessing programs.

Wireless sensor networks (WSNs) is a new intelligent network technology (Raghavendra et al., 2011; Ren et al., 2018), which is composed of a large number of stationary or moving sensor nodes in a self-organized and multi-hop mode, in order to monitor the information of target area and report to the control center by cooperative sensing, acquisition, processing and wireless transmission (Wei et al., 2012; Zhang et al., 2015).

The research on WSNs began at the end of the last century, and it is still at the stage of research. Some systems in the fields of environmental monitoring and smart home are researched and developed (Trab et al., 2013; Hong et al., 2007; Mohd et al., 2011).

The main communication technologies used in WSNs include: Zigbee (Tao et al.,2012; Sung et al.,2009), UWB (Zhang et al., 2009), and Wi-Fi (Patrick et al., 2007), with different features according to the different design purposes. The standard ZigBee is suitable for short distance (tens of meters), low energy consumption and low bandwidth communication, but the network communication distance of PA-Zigbee can exceed 1 kilometer, UWB is suitable for short distance and high bandwidth communication, $\mathrm{Wi}-\mathrm{Fi}$ is suitable for short distance and high bandwidth communication.

According to the characteristics of TPS automatic measurement applications, the PA-Zigbee sensor network is suitable to organize prism nodes (Kuang et al., 2013). In order to adapt to the complex observation environment, solve the problem of visibility impact on communication distance and reliability, we use $470 \mathrm{MHz}$ Zigbee instead of $2.4 \mathrm{GHz}$ Zigbee.

\subsection{On-off Prism Design and Implementation}

On the whole, the prism node consists of an optical measurement section and a wireless sensor control part. It includes a measuring prism, an electrically controlled dimming glass, a control unit, a wireless communication module and a power supply module, etc. The optical measurement section is to set up an electrically controlled dimming glass before a measuring prism. By controlling whether the electrically controlled dimming glass is energized, the glass is in the state of transparency or fuzziness, and realize whether the prism on working state. The wireless sensor control part is responsible for the wireless ad-hoc network and power supply control, that is, the sink node and terminal nodes are organized automatically, and all the terminal prism nodes are controlled by the sink node.

According to the application requirements, the computer control program sends instruction to a prism node by the coordinator node wirelessly, and then control the electronic prism node dimming glass power on or power off, to open or close the transmission state of the prism, and close other prism nodes to avoid interference total station measurement. The dimming glass is transparent when power on, and turn to frosted when power off (Pena et al., 2002; Ju et al., 2017). The principle of on-off prism node is illustrated in Figure 1.

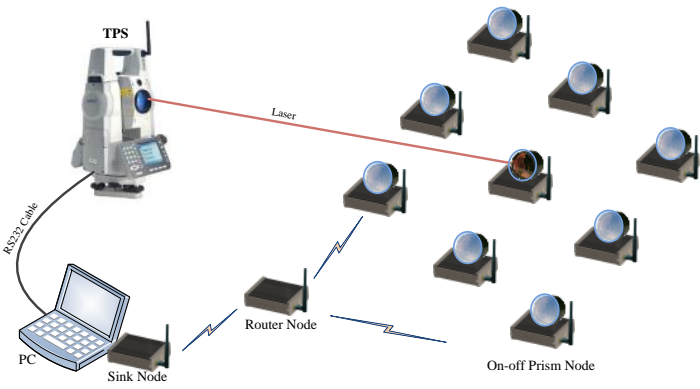

Figure1. TPS working with On-off Prism Node.

\subsubsection{Global Design}

The node is composed of micro processing chip and control circuit, Zigbee communication module, antenna, power supply unit, data interface and indicator light etc. It is used for network communication control, node status control with the embedded program.

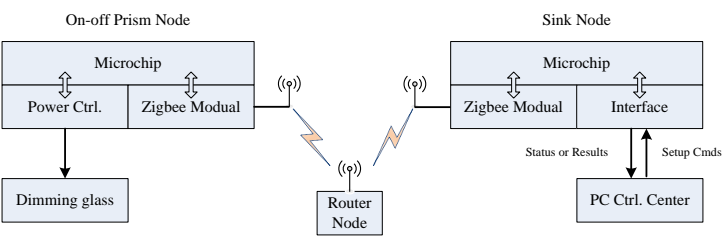

Figure 2. The structure and application diagram of nodes.

The role of the node is divided into 3 kinds: terminal node, route node and sink node. The first kind is on-off prism node. The major function of router node is routing to expand the network. The third is sink node, which is connected with computer or remote communication device. A network has only one sink node, and all data is gathered to this node. The structure and application of nodes are shown in Figure 2.

\subsubsection{Functional Unit Design for On-off Prism Node}

The main functional units are designed as follow.

(1) Power supply unit. It provides other units of the single board a $3.3 \mathrm{~V}$ output, which is convert form the 5 16V DC power input by the power supply.

(2) Main control unit. It contains LPC1768 and the additional circuit, which is used to receive and process data from the Zigbee communication module and sensor, and to monitor and control the whole system.

(3) Data transmission unit. It has a Zigbee module to receive and send the latest control commands and data. Especially, we use FYZB470-M0-L as ZigBee communication module, which is a $470 \mathrm{MHz}$ ZigBee communication module with the features such as far distance communication, good diffraction and strong anti-jamming ability, which can support for automatically network star, tree, mesh and other network topology.

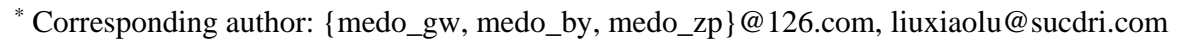


(4) Relay control module. It is assembled to control the dimming glass power off and on.

(5) Booster unit and DC-AC inverter of power are assembled. Because the output voltage supplied by common node is $3.3 \mathrm{~V} \mathrm{DC}$ and the dimming glass needs $48 \mathrm{~V} \mathrm{AC}$, the booster unit and DC-AC inverter of power is essential.

(6) Dimming glass, witch is combined with common prism as a manipulated unit.

Booster unit and DC-AC inverter of power is added on the remote control switch prism node, and they are designed as following.

\section{(a) Booster unit}

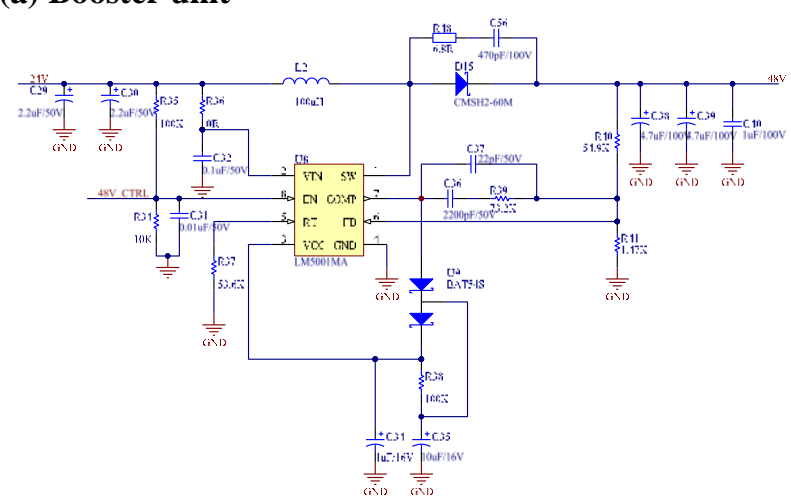

Figure 3. Circuit design diagram of booster unit.

Figure 3 shows the booster unit of transition from $24 \mathrm{~V}$ DC to $48 \mathrm{~V}$ DC. The unit adopts LM5001MA microchip of $\mathrm{TI}$, in which $75 \mathrm{~V} \mathrm{~N}$-type MOS tube is integrated and voltage input at super wide range $3.1-75 \mathrm{~V}$ is used. The first pin SW of LM5001MA can control to close switch, then will cause electricity increase of the inductance. Turn on the switch will prompt the electricity through the diode to the output capacitance. Because of storage from the inductance of the electricity, output capacitance voltage will rise after multiple switch cycle, and the output voltage will be $48 \mathrm{~V}$.

\section{(b) DC-AC Inverter}

The circuit design diagram of DC-AC Inverter is shown in Figure 4. The VOUT+ and VOUT- are output voltage; Q1_1 and Q1_2 are a set of relative SPWM; Q2_1 and Q2_2 are other set of relative SPWM. At the same time, Q1_1 and Q2_2 have same voltage, and Q1_2 and Q2_1 have same voltage in the circuit. If the Q1_1 and Q2_2 are high voltage, two MOSFET of Q4 and Q7 are breakover, meanwhile, Q5 and Q6 are cut-off, electricity will flow from left VOUT to right VOUT; If the Q2_1 and Q1_2 are high voltage, Q5 and Q6 are breakover, meanwhile, Q4 and Q7 are cut-off, electricity will flow from right VOUT to left VOUT. So loop down, $48 \mathrm{v}$ DC is transformed to $48 \mathrm{v}$ AC.

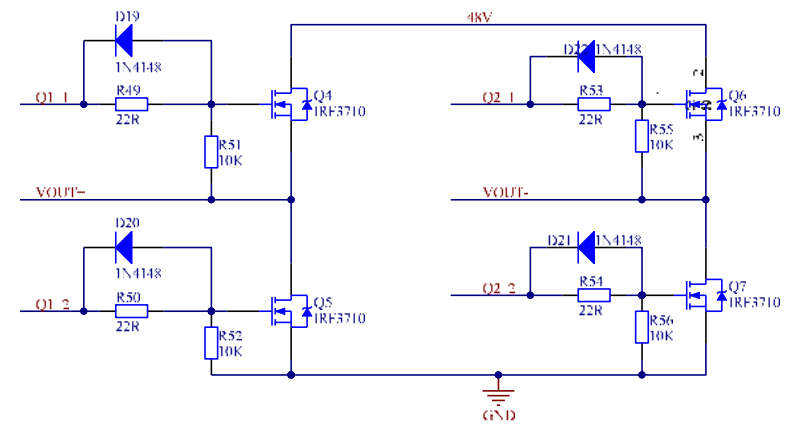

Figure 4. Circuit design diagram of DC-AC Inverter.

\section{Experiment And Analysis}

On-off prism node is shown in Figure 5.

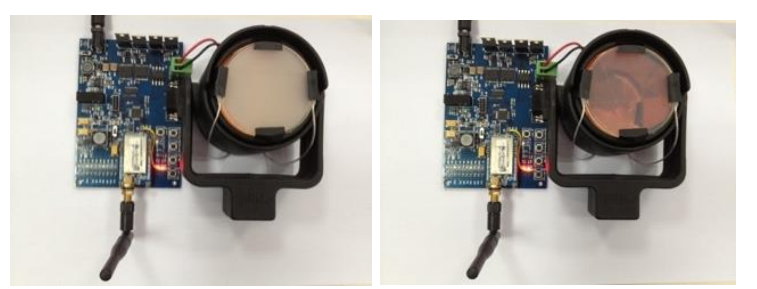

(a) Close status

(b) Open status

Figure 5. Remote control switch prism node.

Figure 5(a) shows the off status of switch prism with power-off of the dimming glass, what means it likes a ground glass. Figure 5(b) shows the on status of switch prism with power-on of the dimming glass, what means it is transparency, and it is available for automatic search of total station.

Due to the limited production conditions, the dimming glass and ordinary prism general are combined to design and implement in this paper. If manufacture is available, the front protect glass of prism can be replaced by dimming glass, and the power line can be outgoing for connecting power-up and -off control node.

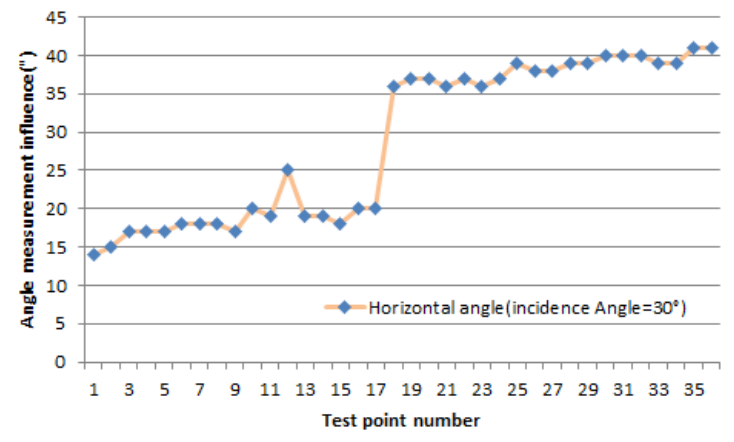

(a) Influence of goniometry with big incidence angle 


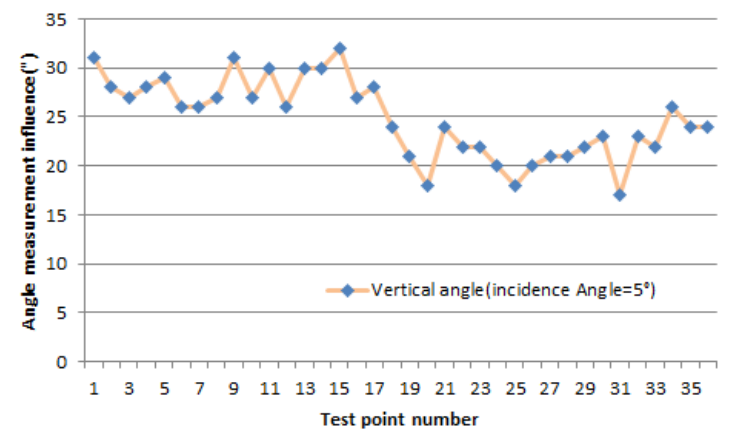

(b) Influence of goniometry with small incidence angle

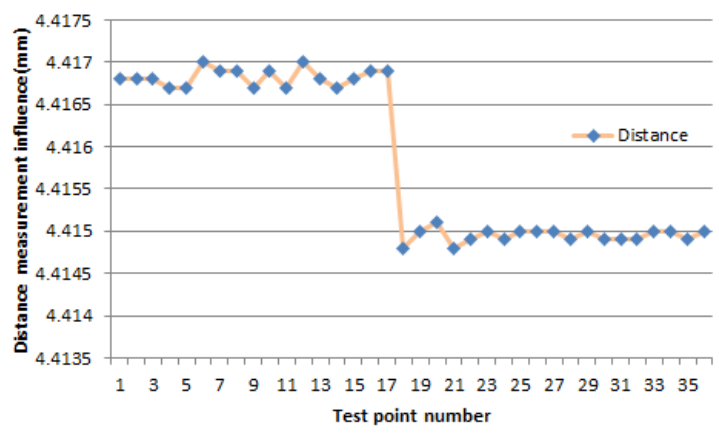

(c) Influence of ranging

Figure 6. Influence of remote control switch prism for accuracy of ranging and goniometry.

The thickness of the dimming glass inevitably has the influence on the accuracy of ranging and goniometry. Through experiment comparison, the values of angle measurement and distance measurement are less affected when the incidence angle is very small; while the values of angle measurement and distance measurement are affected bigger when the incidence angle is increase. As shown in figure 6 , the number $1 \sim 17$ points are switch prism measurement points, and the number $18 \sim 30$ points are normal prism measurement points. At this time, the total station adopts the automatic search mode.

According to the principle of physical optics, the accuracy of goniometry is increased with the increase of glass thickness, and is decreased with the increase of distance measurement. The accuracy of ranging is increased with the increase of glass thickness.

Due to the effect of dimming glass layer on accuracy of angle measurement and distance measurement is very small when TPS optical path of measurement vertical incident prism. So as long as the prism node is installed directly towards the direction of the TPS station, the measurement error can be reduced to a minimum, and the accuracy of result is hardly affected.

\section{Conclusions}

Organize wireless network and control on-off prism nodes based on WSNs can solve the problem of multiple prisms automatic search in a narrow field angle during total station monitoring applications. The device has small power consumption, can be powered by battery, without active power supply. It can work in wireless communication mode, suitable for convenient deployment and flexible control.

Next work goal is continuing to improve the control function of prism node, to make total station monitoring system organize on-off prism nodes more effectively based on WSNs. Control prism nodes with the progress of TPS observing to recognize multiple prisms effectively and automatic point search and initial position learning in deformation applications.

\section{Appendix}

\section{Electronically controlled dimming glass working principle}

The manufacturing method of electronically controlled dimming glass is a layer of electrically controlled PVB interlayer sandwiched in the middle of the float glass, to make the glass transparent or opaque through the control of power supply.

Electronically controlled dimming glass is a branch of smart glass, which was invented by researchers at the Kent State University in the late 80 s of the last century. The principle of dimming glass is illustrated in the following diagram.
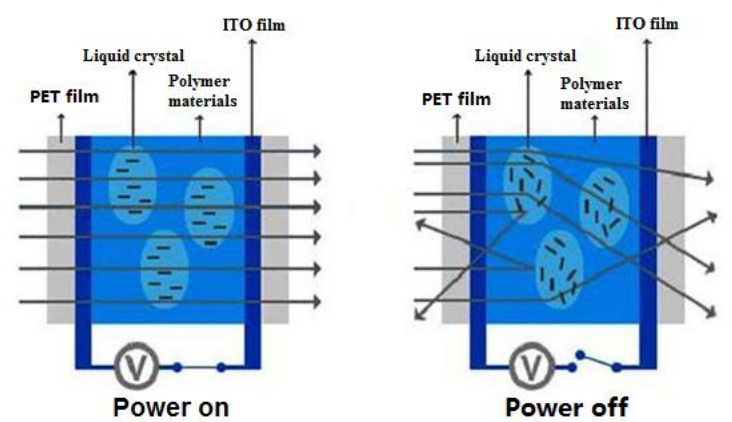

(1) When the dimming glass power off, liquid crystal polymer materials between glass disorderly arrangement, so that the light can not penetrate the glass, then it is milky white opaque state.

(2) When the glass is energized, in the electric field the liquid crystal polymer material between glass ordered arrangement, so the light can pass through the glass, then it is transparent and colorless glass state.

\section{References}

1. Wang, Z., Wan, G., Liu, Y., Ge, Z., 2017. The design and implementation of roller's calibration system in steel plant based on total station. 13th IEEE International Conference on Electronic Measurement \& Instruments (ICEMI), 56-62.

2. Paraforos, D.S., Reutemann, M., Sharipov, G., Werner, R., Griepentrog, H.W., 2017. Total station data assessment using an industrial robotic arm for dynamic 3D in-field positioning with sub-centimetre accuracy. Computers and Electronics in Agriculture 136, 166-175.

3. Gumilar, I., Fattah, A., Abidin, H.Z., Sadarviana, V., Putri, N.S.E., 2017. Landslide monitoring using 
terrestrial laser scanner and robotic total station in Rancabali, West Java (Indonesia). AIP Conference Proceedings, 1857, 060001 (6 pp.).

4. Xiong, H., He, P., Can, Y., Shen, C., 2016. Automatic Target Recognition and Sighting Technology of Total Station. Optics \& Optoelectronic Technology 14, 34-39. STE

5. Raghavendra, V.K., Anna, F., Ganesh, K.V., 2011. Computational Intelligence in Wireless Sensor Networks: A Survey. Communications Surveys \& Tutorials. IEEE 13, 68-96.

6. Ren, Z., Zhang, G., Lin, D., Zhang, Z., Zhao, X., 2018. Review on application of WSNs. Transducer and Microsystem Technologies 37, 1-3.

7. Wei, Y., Mao, Y., Yu, Q., Li, N., 2012. A Structure of Large Scale WSN Based Safety Monitoring System for Natural Gas Industry. Advanced Materials Research, 512-515(4), 2482-2488.

8. Zhang, F., Song X., Liu J., 2015. Data Acquisition and Intelligent Management System of Foundry Shop Based on WSNs. Foundry Technology 36, 785-

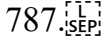

9. Trab, S., Zouinkhi, A., Boussaid, B., Abdelkrim, M., N., 2013. Application of distributed fault detection in WSN to dangerous chemical products based on Bayesian approach. International Conference on Advanced Logistics and Transport (ICALT 2013), 129-134.

10. Hong, W.P., Dunford, W., 2007. Networked Intelligent Control System using Smart MCC \& Lonworks/Ethernet Server for Operating Multiinduction Motor of Industrial Field. Proceedings of the IASTED International Conference on Communication Systems, Networks, and Applications, CSNA 2007, 12-18.

11. Mohd, I.M.R., Adnan, A.A., 2011. Development of Intelligent Wireless Sensor Networks for Human Comfort Index Measurement. Procedia Computer Science 5, 232-239.

12. Tao, W., Chen, X., Jia, Z., Qian Z., 2012. Construction of a WSN Teaching Experimental Platform Based on ZigBee. Research and Exploration in Laboratory 31, 231-234.

13. Sung, W.T., Hsu, Y.C., 2009. Designing an Industrial Real-time Measurement and Monitoring System based on Embedded System and ZigBee. Expert Systems with Applications 38, 4522-4529.

14. Zhang, J., Orlik, P.V., Sahinoglu, Z., 2009. UWB Systems for Wireless Sensor Networks. Proceedings of the IEEE 97,313-331.

15. Patrick, M.C., Ira, S., 2007. Wi-Fi... Why Now? Exploring New Wireless Technologies for Industrial Applications. Proceedings of the International Instrumentation Symposium, 625-635.

16. Kuang, Y.L., Sung, J.H., Wen, T.S., 2013. Intelligent Multi-sensor Control System based on Innovative Technology Integration via ZigBee and Wi-Fi
Networks. Journal of Network and Computer Applications 36,756-767.

17. Ju, C., Sun, H., Wang, L., Hu, X., Li, C., 2017. Research Progress on Smart Windows Based on Polymer Liquid Crystal. Reinder, C., Zhou, G., 2017. Journal of South China Normal University (Natural Science Edition) 49, 21-25.

18. Pena, J.M.S., Vazquez, C., Perez, I., 2002. Electrooptic system for online light transmission control of polymer dispersed liquid crystal windows. Optical Engineering 41, 1608-1611. 